\title{
SEGURANÇA EM BANCO DE DADOS
}

Thaynára da Silva, Paulo Roberto Rosa

Instituto Federal de Educação, Ciência e Tecnologia de São Paulo - IFSP, Curso Superior de Tecnologia em Análise e Desenvolvimento de Sistemas, Campus de Presidente Epitácio, SP. E-mail: thaynara-r@hotmail.com

\section{RESUMO}

O presente artigo tem como objetivo apresentar a importância da segurança em banco de dados para as organizações uma vez que as empresas têm se tornado cada vez mais dependentes desse tipo de tecnologia que tem um papel fundamental em razão do armazenamento de dados e informações valiosas. Serão abordadas algumas medidas para controle de acesso e formas de ataques visto que o numero de ataques ao banco de dados tem aumentado por ser o coração de qualquer organização. A metodologia utilizada foi baseada em pesquisas bibliográficas em livros da área de banco de dados e segurança da informação. Conclui-se que este tipo de tecnologia tem função significativa por conter informações tão sigilosas e por ser o diferencial para uma empresa manter-se competitiva no mercado, portanto a segurança tem um papel de extrema importância para que isto aconteça.

Palavras-chave: Banco de Dados. Segurança. Medidas de Controle. Informação. Injeção SQL.

\section{DATABASE SECURITY}

\section{ABSTRACT}

The present article has the aim of presenting the importance of database security for the organisations once the companies have become even more dependent of this type of technology that has a fundamental role for the reason of storage of data and valuable information. Some measures of access control and methods of attacks will be approached as the number of database attacks has increased for being the heart of any organisation. The methodology used was based on bibliographic research in books of database and information security area. It is concluded that this type of technology has a significant role for containing secret information and for being the differential for a company to maintain competitive on the market, therefore the security has an extremely important role for this to happen.

Keywords: Database. Security. Measures of Control. Information. SQL Injection. 


\section{INTRODUÇÃO}

No mundo contemporâneo as empresas necessitam captar, processar, armazenar e disseminar um volume crescente de informações. Para que isso seja possível, elas lançam mão de uma série de tecnologias que visam auxiliá-la nesse trabalho, sendo os bancos de dados um exemplo de tecnologia para tal finalidade. No que se refere a esse tipo específico de tecnologia, este artigo buscará esclarecer questões relacionadas a segurança em banco de dados, tais como: o que é segurança em banco de dados? Qual é a importância da segurança em banco de dados para uma empresa?

Parte-se do princípio que segurança em banco de dados seja a proteção de informações valiosas de uma empresa. Imagina-se que essa proteção seja contra defeitos lógicos ou de hardware, bem como a acesso e manipulação não autorizados. Presume-se que a importância da segurança em banco de dados reside em manter as informações seguras e a competitividade no mercado.

A importância das informações no contexto atual é tamanha que elas tanto podem representar uma fonte de vantagem competitiva para a organização quanto tem potencial para, se mal utilizadas ou mal protegidas, tornarem-se fontes de vulnerabilidades. Por isso é importante que os profissionais da área de TI saibam dos possíveis riscos aos quais pode estar exposto um banco de dados no dia a dia. Dominar formas eficientes de proteger esses dados pode evitar prejuízos tanto para a empresa como para seus clientes e usuários.

O objetivo deste artigo é ressaltar a importância da segurança em banco de dados, já que as informações empresariais, a depender do seu grau de sensibilidade, podem ser usadas tanto a favor quanto contra a empresa. Portanto, para que uma empresa se mantenha competitiva é necessário ter as informações bem protegidas.

\section{METODOLOGIA}

Este artigo foi desenvolvido mediante uma abordagem de caráter qualitativo, com a utilização de fontes de dados secundárias. Serão realizadas pesquisas bibliográficas baseadas em livros da área de banco de dados, segurança da informação e outras publicações correlatas.

\section{BANCO DE DADOS}

No passado, as empresas armazenavam as informações em arquivos físicos, o que causava grande acúmulo de papel e dificultava a organização, atualização e acesso a essas informações. Porém, com o surgimento e evolução das tecnologias computacionais, as empresas passaram a investir na aquisição de computadores e as informações passaram a ser armazenadas em bancos de dados digitais. De acordo com Date (2003, p. 10), "um banco de dados é uma coleção de dados persistentes, usada pelos sistemas de aplicação de uma determinada empresa".

Um banco de dados apresenta algumas propriedades através das quais indica que sua abrangência vai muito além de uma mera coleção de dados. A primeira propriedade de um banco de dados refere-se a este ser um conjunto lógico e ordenado de dados com significado. Não será considerado banco de dados uma coleção de dados aleatória, sem uma finalidade ou objetivo (MACHADO, 2008).

Construído e povoado com dados, o banco de dados possui um determinado objetivo e simboliza um minimundo ou universo de discurso onde são consideradas as propriedades de objetos que interessam aos usuários para fins de processamento computacional (ELMASRI; NAVATHE, 2011). Um banco de dados tem tamanho e complexidade distintas. O celular é um exemplo de device que pode conter um ou mais banco de dados de pequeno porte e baixa complexidade. Como exemplos de grandes bancos de dados podem ser considerados os da Amazon.com e Alibaba.com, empresas multinacionais com atuação mundializada e que comercializam produtos - livros, jogos, eletrônicos, roupas etc. - provenientes de diversos pontos 
do planeta junto a consumidores igualmente distribuídos ao redor do globo terrestre.

\section{SISTEMA GERENCIADOR DE BANCO DE DADOS (SGBD)}

Como desdobramento evolutivo da tecnologia de banco de dados surge o sistema gerenciador de banco de dados (SGBD) ou Database Management System (DMS). O SGBD é uma composição de softwares responsáveis pelo gerenciamento do banco dados. De acordo com Alves (2009, p. 23), "um sistema de gerenciamento de banco de dados (SGBD) é uma coleção de ferramentas e programas que permitem aos usuários a criação e manutenção do próprio banco de dados". Exemplos de SGBDs mais comuns são: Oracle, MySQL, PostgreSQL e SQL Server. Um SGBD facilita o processo de definição, construção, manipulação e compartilhamento do banco de dados e aplicações.

Um SGBD contém, dentre suas funções significativas, um subsistema de segurança que protege o banco de dados contra acessos não autorizados. O Administrador do Banco de Dados ou Database Administrator (DBA) utiliza esse subsistema para decidir quais usuários podem acessar o banco de dados e os tipos de operações que poderão efetuar, por exemplo: ler, atualizar, adicionar e/ou apagar (ELMASRI; NAVATHE, 2011).

O DBA é responsável pela segurança geral de um sistema de banco de dados. Ele é a autoridade máxima e utiliza uma conta conhecida como superusuário ou conta do sistema para desempenhar suas funções como, por exemplo, decidir os privilégios dos usuários. Segundo Ramakrishnan \& Gehrke (2008, p. 18), “o DBA é responsável por assegurar que o acesso não autorizado aos dados não seja permitido. Em geral, nem todos devem ser capazes de acessar todos os dados".

\section{SEGURANÇA DA INFORMAÇÃO}

Um banco de dados pode conter informações muito valiosas para a empresa ou até mesmo para as pessoas. A divulgação de informações não autorizadas pode afetar a empresa de diversas formas, como levar a perda de clientes ou de mercado, ou até mesmo a ações judiciais. A segurança da informação, segundo Alves (2006, p. 1), “[...] visa proteger a informação de forma a garantir continuidade dos negócios, minimizando os danos e maximizando o retorno dos investimentos e as oportunidades de negócios".

Há alguns atributos significativos relacionados à segurança da informação que, se aplicados de forma correta, ajudam na proteção das informações. Considera-se necessário que a empresa compreenda-os para preparar-se e fazer frente a eventuais problemas que possam ocorrer, conhecer a origem, a extensão e a melhor maneira de tratar os riscos, de acordo com a situação e as possibilidades da empresa.

O primeiro destes atributos é a integridade, que tem intuito de garantir que a informação mantenha todas as características originais. A informação só é considerada integra quando não sofre nenhuma modificação - intencional ou acidental - ou quando essas modificações são autorizadas e realizadas de forma controlada (ELMASRI; NAVATHE, 2011). Por exemplo, os alunos podem ver suas notas, mas não podem alterá-las. O segundo atributo é a disponibilidade e objetiva garantir que as informações estejam disponíveis e acessíveis sempre que necessário. De acordo com Sêmola (2003, p. 45), "toda informação gerada ou adquirida por um individuo ou instituição deve estar disponível aos seus usuários no momento em que os mesmos delas necessitem para qualquer finalidade".

O terceiro atributo é a confidencialidade e visa certificar que somente pessoas autorizadas terão acesso à determinada informação. A intenção é que somente o destinatário a receber a mensagem tenha acesso às informações que ela contém. Segundo Sêmola (2003, p. 45), "toda informação deve ser protegida de acordo com o grau de sigilo de seu conteúdo, visando a limitação de seu acesso e uso apenas às pessoas para quem elas são destinadas". A característica 
do quarto atributo, a autenticidade, é garantir que a informação é autêntica, ou seja, verdadeira. De acordo com a Norma ISO/IEC 17799 apud Alves (2006, p. 2) refere-se a "'necessidade de verificar que uma comunicação, transação ou acesso a algum serviço é legítimo'". O quinto atributo é não-repúdio e tem como característica assegurar que uma pessoa não consiga negar a autoria de uma mensagem, informação, ato ou documento (ISO/IEC 17799 apud ALVES, 2006). Só pode garantir o não-repúdio quando houver integridade e autenticidade, quando for possível determinar quem enviou a mensagem e quando a mensagem não foi alterada. E por fim, a conformidade, o sexto atributo, procura certificar que os sistemas e processos que originaram a informação atendem aos preceitos legais, regulamentares, contratuais e corporativos (ISO/IEC 17799 apud ALVES, 2006).

\section{SEGURANÇA EM BANCO DE DADOS}

A segurança em banco de dados abrange a proteção dos dados contra roubo, destruição mal-intencionada, atualização não autorizada, entre outros. Portanto, torna-se significativa para a empresa, uma vez que sua situação pode ser drasticamente afetada por qualquer vulnerabilidade na segurança de um banco de dados. No que se refere a segurança de banco de dados, algumas medidas para controle de acesso e ataques aos bancos de dados são necessárias.

Para proteger o banco de dados contra ameaças, medidas de controle de acesso devem garantir a inviolabilidade de alguns atributos de segurança da informação já citados anteriormente, quais sejam: integridade, disponibilidade e confidencialidade. A esses devem ser adicionados o próprio controle de acesso em si e a criptografia de dados.

O controle de acesso é uma das principais medidas para manter a segurança de um banco de dados e fica sob a responsabilidade de um DBA. Impedir que pessoas não autorizadas tenham acesso aos sistemas tornou-se um desafio a ser superado pelas empresas. Elmasri e Navathe (2011, p. 564) afirmam que

O mecanismo de segurança de um SGBD precisa incluir provisões para restringir o acesso ao sistema de banco de dados como um todo. Essa função, chamada de controle de acesso, é tratada criando-se contas do usuário e senhas para controlar o processo de login pelo SGBD.

Quando uma pessoa ou um grupo de pessoas precisa acessar um banco de dados é necessário que se faça a requisição de uma conta de usuário. Logo, o DBA decide se há necessidade de criação de conta para essa pessoa ou esse grupo de pessoas (ELMASRI; NAVATHE, 2011).

A criptografia de dados, segundo Ramakrishnan e Gehrke (2008, p. 590), refere-se à aplicação de "[...] um algoritmo [...], usando uma chave de criptografia especificada pelo usuário ou pelo administrador do banco de dados". É uma das melhores soluções para se armazenar ou transferir dados com segurança. Em caso de invasão ou acesso não autorizado e estando os dados criptografados, haverá dificuldades para decifrar o real significado das informações, uma vez que a criptografia possibilita a sua compreensão apenas por pessoas previamente autorizadas. Segundo Elmasri e Navathe (2011, p. 564), apenas "[...] os usuários autorizados recebem algoritmos de codificação ou decodificação (ou chaves) para decifrar os dados".

Para ter acesso aos dados originais após a criptografia dos dados é necessário a aplicação de um algoritmo de descriptografia. De acordo com Ramakrishnan e Gehrke (2008, p. 590), "sem a chave de descriptografia correta, o algoritmo de descriptografia produz lixo". A chave para tornar as informações compreensíveis depende da estratégia de criptografia utilizada, sendo possível a utilização de criptografia com chave simétrica ou pública.

Dada a importância e centralidade das informações para as empresas na realidade contemporânea, os bancos de dados, por armazenarem dados e informações cruciais ao negócio, 
tem sua potencialidade para ser alvo de ataques maximizada. Dentre os vários tipos de ataque possíveis, merecem destaque aqui o abuso de privilégio e a injeção de SQL (SQL Injection). O abuso de privilégio ocorre quando o usuário tira proveito das permissões que lhe foram concedidas para realizar operações as quais não está autorizado, o que é exemplificado por Elmasri e Navathe (2011, p. 575) em um caso hipotético onde "[...] um administrador que tem permissão para alterar a informação do aluno pode usar esse privilégio para atualizar notas de alunos sem a permissão do instrutor".

A metodologia de ataque denominada injeção de SQL pode apresentar-se de duas formas: manipulação (modificação) de uma instrução SQL já existente ou injeção de uma nova instrução SQL. A injeção de SQL funciona com a inserção de comandos SQL através de formulários web, comandos que podem ser de manipulação de dados, tais como select, insert, update e delete, ou então para definição de dados, tais como create, drop e alter. Segundo o PHP Group, a

Injeção direta de comandos SQL é uma técnica onde um atacante cria ou altera comandos SQL existentes para expor dados escondidos, ou sobrescrever dados valiosos, ou ainda executar comandos de sistema perigosos no servidor. Isso é possível se a aplicação pegar a entrada do usuário e combinar com parâmetros estáticos para montar uma consulta SQL.

A pessoa mal-intencionada pode tirar proveito de uma vulnerabilidade do código implementado no banco de dados para realizar o ataque. Para Elmasri e Navathe (2011, p. 576) "em um ataque de Injeção de SQL, o atacante injeta uma entrada de cadeia de caracteres pela aplicação, que muda ou manipula a instrução SQL para o proveito do atacante". O método mais comum refere-se à manipulação de SQL, que funciona com à alteração de um comando SQL. A inserção de condições à cláusula "where" de uma consulta SQL seria um exemplo desse tipo de ataque, como apresentam Elmasri e Navathe (2011, p. 576, grifo do autor):

SELECT * FROM usuário WHERE nomeusuario = 'jaque' and SENHA = 'senhajaque'. O atacante pode tentar alterar (ou manipular) a instrução SQL, alterando-a da seguinte forma: SELECT * FROM users WHERE nomeusuario $=$ 'jaque' and $($ SENHA $=$ 'senhajaque' or ' $x$ ' $=$ ' $x$ ' $)$.

Neste exemplo de manipulação de SQL, que acontece durante o login no banco de dados, com a modificação o invasor consegue fazer o login sem saber a senha do usuário e terá condições para realizar as mesmas operações que o usuário devidamente autorizado tem permissão para fazer, o que pode trazer danos à empresa.

\section{CONSIDERAÇÕES FINAIS}

Pelas análises realizadas entende-se que a segurança em banco de dados é de extrema importância, visto que armazena dados e informações cruciais para a empresa. Por abrigar dados e informações com diferentes graus de sensibilidade, manter a segurança em um banco de dados é uma atividade complexa e necessária. Um banco de dados pode conter informações muito valiosas e qualquer vulnerabilidade pode resultar em grande impacto e afetar drasticamente a continuidade dos negócios.

Nesta perspectiva Laudon e Laudon (2007) apresentam o caso envolvendo as empresas Mastercard Internacional, Visa, American Express e Discover - ocorrido em 2005. O caso envolveu a exposição indevida de informações confidenciais dos clientes devido a uma falha de segurança na empresa Cardsystems Solutions, responsável pelo processamento dos pagamentos. Explorando vulnerabilidades presentes na rede da Cardsystems, um invasor conseguiu obter informações dos titulares de cartões mediante a introdução de um script ou programa de computador especificamente para esta finalidade. 
O armazenamento de dados pela Cardsystems ao menos com a adoção de medidas básicas de segurança - como por exemplo a criptografia - poderiam ter evitado o incidente e/ou então a interpretação dos dados pelo invasor. A falha de segurança afetou sobremaneira a imagem e credibilidade da Cardsystems, o que, além de conduzir ao encerramento do vínculo contratual pelas empresas Matercard Internacional e Visa, afetou significativamente os resultados ao ponto de inviabilizar o negócio (LAUDON; LAUDON, 2007). Este episódio torna evidente a importância da segurança da informação, incluindo a segurança do banco de dados, para fazer frente a possibilidade de que, com conhecimentos técnicos específicos e a ausência de mecanismos adequados de segurança, invasores consigam acessar bancos de dados de empresas. A execução da sequência correta de comandos torna possível explorar as vulnerabilidades presentes em uma rede e/ou banco de dados.

Diante do exposto, por conter informações sigilosas, é importante que a empresa tome todas as medidas necessárias à manutenção da segurança de seu banco de dados e assim evitar danos que podem ser irreparáveis. Dentre as medidas básicas destinadas à proteção do banco de dados destacam-se o controle de acesso e a criptografia dos dados. Cautela é a palavra de ordem do DBA no momento de conceder privilégios aos usuários, pois assim evitará outorgar privilégios indevidos a usuários e atuará de forma preventiva contra esse tipo de ataque.

O armazenamento de dados e informações que podem agregar muito valor aos negócios aumenta a potencialidade do banco de dados tornar-se alvo de ataques. Por essa razão, a empresa deve primar por uma atuação proativa e pela adoção de medidas preventiva, bem como efetuar todas as validações possíveis para evitar ataques. Várias medidas e técnicas podem ser empregadas pela empresa para proteger seu banco de dados, como por exemplo a implementação de variáveis de ligação e a filtragem de entrada.

Compreende-se, assim, que a proteção das informações tornou-se condição sine qua non à manutenção da competitividade organizacional. Por essa razão, a segurança em banco de dados jamais deve ser preterida pela empresa, visto que os bancos de dados, por conterem dados e informações que podem agregar valor ao negócio e propiciar as condições necessárias à obtenção do diferencial e da vantagem competitiva, tiveram maximizadas as potencialidades para tornaremse alvo de ataques.

\section{REFERÊNCIAS}

ALVES, Gustavo A. Segurança da informação: uma visão inovadora da gestão. Rio de Janeiro: Ciência Moderna, 2006.

ALVES, William P. Banco de dados: teoria e desenvolvimento. 1. ed. São Paulo: Érica, 2009.

DATE, Christopher J. Introdução a sistemas de banco de dados. 8. ed. Rio de Janeiro: Elsevier, 2003.

ELMASRI, Ramez; NAVATHE, Shamkant B. Sistemas de banco de dados. 6. ed. São Paulo: Pearson, 2011.

LAUDON, Kenneth C.; LAUDON, Jane P. Sistemas de informações gerenciais. 7. ed., São Paulo: Pearson, 2007.

MACHADO, Felipe N. R. Banco de Dados: projeto e implementação. 2. ed. São Paulo: Érica, 2008.

PHP, Group. Manual do PHP: Injeção de SQL. Disponível em:

<http://php.net/manual/pt_BR/security.database.sql-injection.php>. Acesso em: 28 nov. 2016. 
RAMAKRISHNAN, Raghu; GEHRKE, Johannes. Sistemas de gerenciamento de banco de dados. 3 . ed. São Paulo: McGraw-Hill, 2008.

SÊMOLA, Marcos. Gestão da segurança da informação: uma visão executiva. Rio de Janeiro: Elsevier, 2003. 